\title{
Model-free Subspace Based Dynamic Control of Mechanical Manipulators
}

\author{
Muhammad Saad Saleem and Ibrahim A. Sultan \\ University of Ballarat \\ Australia
}

\section{Introduction}

Realtime identification and dynamic control of mechanical manipulators is important in robotics especially in the presence of varying loading conditions and exogenous disturbances as it can affect the dynamic model of the system. Model free control promises to handle such problems and provides solution in an elegant framework. Model free control has been an active area of research for controlling plants which are difficult to model and time varying in nature.

The proposed framework takes the objective in operational space. Benefit of specifying objective in operational space along with direct adaptive control is self evident. In this framework, subspace algorithm is used for model identification. $\mathscr{H}_{\infty}$ is used for robust control of manipulator dynamics. Because of the seamless integration of identification and control modules, explicit values of dynamic parameters is not calculated. The model free control system is capable of explicitly incorporating uncertainties using $\mu$-synthesis. Uncertainty models can be calculated from experimental data using model unfalsification. The proposed control system employs a black box approach for dynamics of mechanical systems. The chapter also presents results from a simulation of a planar robot using MATLAB $\AA$ and Simulink $®$ from MathWorks Inc.

\subsection{Notations}

The rigid body model adopted in this chapter is given by

$$
M(q) \ddot{q}+C(q, \dot{q})+G(q)+\xi(q, \dot{q})=u,
$$

where $M(q)$ is the inertia tensor matrix, $C(q, \dot{q})$ is the Coriolis and centripetal forces, $G(q)$ is gravity, and $\xi(q, \dot{q})$ denotes unmodeled non-linearities. Joint variables, their velocities, and positions are donated by $q, \dot{q}, \ddot{q} \in \Re^{n}$. In case of revolute joint, $q$ is the angle while in prismatic joint, $q$ represents the distance. The torques generated by actuators are represented by $u \in \Re^{n}$. It is assumed that the mechanical manipulator is fully actuated, non-redundant and the Jacobian is known. If the position of endeffector is given by forward kinematics equation i.e. $x=f_{\text {kinematics }}(q)$. It can be differentiated by $\partial q$ to obtain

$$
\dot{x}=J(q) \dot{q}, \ddot{x}=J(q) \ddot{q}+\dot{J}(q) \dot{q},
$$

Source: Robotics, Automation and Control, Book edited by: Pavla Pecherková, Miroslav Flídr and Jindřich Duník, ISBN 978-953-7619-18-3, pp. 494, October 2008, I-Tech, Vienna, Austria 


\subsection{Problem statement}

The problem under discussion can be stated as

Given a trajectory of end-effector in operational space $x, \dot{x}, \ddot{x} \in$ $\Re^{n}$, joint variables $q, \dot{q}, \ddot{q} \in \Re^{n}$, and forward kinematics function $f_{\text {kinematics }}(\bullet)$. Find actuator torque $u \in \Re^{n}$ such that the endeffector smoothly follows the trajectory in the presence of gravity and exogenous disturbances and provides stable solution near singularities.

This problem is the amalgamation of inverse dynamics in which $M(q), C(q, \dot{q})$, and $G(q, \dot{q})$ are known i.e. $u=f(q, \dot{q}, \ddot{q})$, dynamic parameter identification in which $M(q), C(q, \dot{q})$, and $G(q, \dot{q})$ are calculated, and robust control to cater unmodeled non-linearities and disturbances in the system.

\section{Control of articulated manipulators}

Control of articulated manipulators can be divided into two main categories:

- Joint space control

- Operational space control

Joint space control is consisted of two subproblems. First, manipulator inverse kinematics is performed and then joint space control scheme is devised to allow the end effector to follow a reference input. The main computational burden in this scheme is incurred by the inverse kinematics procedure, which is normally performed by using different optimization techniques; particularly in redundant systems where there can be infinite solutions for a given task (Kim et al. (2003)). Many implementations of joint space control can be found in the literature (Laib (2000); Kelly (1997); Arimoto (1995); Kelly (1993); Wen et al. (1992); Tomei (1991); Takegaki \& Arimoto (1981); Zhang et al. (2000)).

In many applications, the desired path of end effector is specified in the operational space (e.g. Cartesian frame). Operational space control, on the other hand, has also been used for constrained manipulator motions (Sapio \& Khatib (2005)). These constraints can be because of gravity, or kinematically imposed. It can be seen in Figure 2 that inverse kinematics is embedded in the closed-loop control law but not explicitly performed as shown in Figure 1 (Sciavicco \& Siciliano (2000)). Operational space control and task space control allude to the same concept (Xie (2003)).

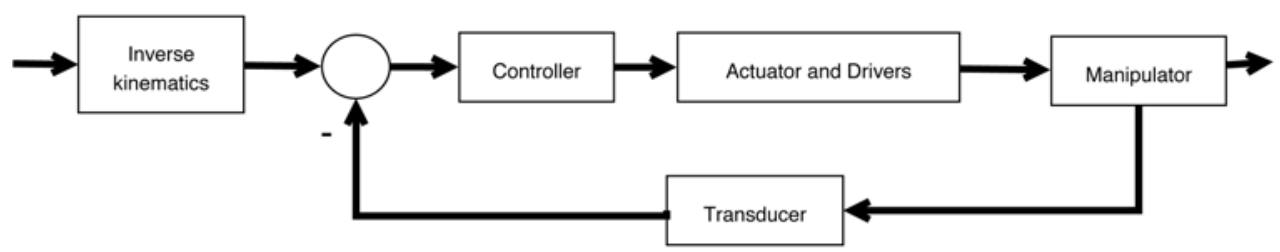

Figure 1. Joint space control

The proposed architecture controls the manipulator in joint space. The reason behind is the very fact that inverse kinematics is highly non-linear in nature. It is assumed the analytical 
Jacobian $J_{A}$ is available, the manipulator is fully actuated and non-redundant. Numerical solutions to inverse kinematics are more complex and are manipulator specific (Khalil \& Dombre (2004)). Numerical based inverse kinematics is out-of-scope of this chapter.

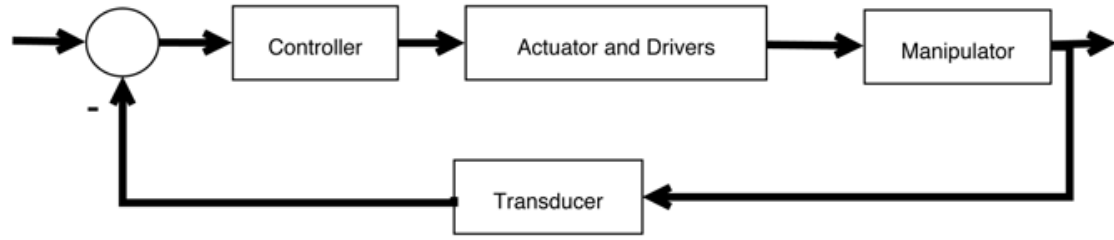

Figure 2. Operational space control

\section{Model free control}

There are four methods to use experimental data as shown in Table 3 (Woodley (2001)). Mainly, choice depends on application. For realtime systems which are fairly easy to model, indirect control is a better choice. The system then adapts itself and updates its model parameters according to the conditions gathered from the measured data. Normally an online model-based design is referred as indirect control. If a system is hard to model from first principles (e.g. Newton's laws of motion) or there are time varying nonlinearities then a direct adaptive control would suit the application. Examples of plants which are difficult to model are arc furnaces (Wilson (1997)) and helicopter rotors (Lohar (2000)). Biped robots on the other hand can be modeled but they exhibit time varying nonlinearities (Wolkotte (2003); Kim et al. (2004); Caballero et al. (2004)).

\begin{tabular}{|c||c|c|}
\hline & With Plant Model & Without Plant Model \\
\hline \hline Online & Indirect Adaptive & Direct Adaptive \\
\hline Offline & Model Based Design & Direct Control Design \\
\hline
\end{tabular}

Table 1. Four different techniques of control design from experimental data

\subsection{General predictive control}

Model free comes under the category of "general predictive control" (GPC). Model free implementations range from fuzzy and neural control (Boyd \& Little (2000); Cheng (2004)) to crisp control techniques (Favoreel et al. (1999a); Woodley et al. (2001a)). However, crisp control is regarded as reliable and explicitly defines performance objective when compared with fuzzy control techniques (Athans (1999)).

In direct adaptive control techniques, an explicit model formation is not needed; this is why it is referred to as model free control. Plant input and output values are observed in realtime, and a controller is designed for the estimated plant model. Model free is actually a misnomer, as the data from the plant's input and output also represent some kind of plant information. In a model free control implementation, the system identification and the controller synthesis techniques are seamlessly integrated to reduce the computational burden, which makes it more suitable for realtime applications. Predictive control has been applied with $\mathscr{H}_{\infty}$ optimal predictors and $\mathscr{H}_{2}$ cost functions (Grimble (1998)), $\mathscr{H}_{\infty}$ optimal predictors and $\mathscr{H}_{\infty}$ control costs (Zhao \& Bentsman (1999)), and mixed $\mathscr{H}_{2} / \mathscr{H}_{\infty}$ minimax predictors (Tse et al. (1993)). Subspace predictors have also been used for direct control with 
quadratic (Favoreel et al. (1998, 1999b,a)) and $\mathscr{H}_{\infty}$ cost functions (Woodley et al. (2001b); Woodley (2001); Woodley et al. (2001a)). Other implementations include adaptive inverse control (Widrow \& Walach (1994)), LMS1 (Widrow \& Stearns (1985)), FxLMS2 and its alternatives (Sayyar-Rodsari et al. (1998)), identification and control based on the $\left(\mathscr{H}_{\infty}\right)$ loop shaping (Date \& Lanzon (2004)), and Lyapunov-based framework (Haddad et al. (2003); Hayakawa et al. (2004)).

$\mathscr{H}_{\infty}$ is famous amongst control engineers because of its ability to control $\mathrm{MIMO}^{3}$ systems built on strong mathematical foundations.

\subsection{System identification}

System identification is used to build dynamical models from measured input-outpu data of a plant. There are many system identification techniques. The list starts with the classical prediction error (PE) and its variants; the auto regression with exogenous input (ARX), output error (OE), auto regression moving average with exogenous input (ARMAX), and Box Jenkins (BJ) (Norton (1986); Ljung (1999)). Subspace identification methods (SIM) have many advantages over classical system identification techniques (Overschee \& Moor (1996)). Notables are;

- From plant's input and output data, a predictor is found. This bears similarity to the Kalman filter states, and transforms the analysis into a simple least square problem. As such, the whole architecture could be streamlined and in a user-friendly fashion.

- When implemented in direct adaptive control, the plant model does not require simplification. Simplification or model reduction can omit useful information. Instead, in subspace identification methods all the plant information is stored in a compact form of a subspace predictor.

- The output of subspace identification methods can be in the state space form which makes it easy to implement on a computer but its architecture has been exploited in different model free implementations as well (Woodley 2001b, Favoreel 1999a).

Wernholt used SIM to solve system identification problem for an ABB IRB 6600 robot (Wernholt (2004)). Hsu et al. used N4SID in style translation for human motion (Hsu et al. (2005)). These are some of the examples that show how SIMs are being used.

\subsubsection{Reported problems in subspace identification methods}

There are a few problems in subspace identification methods. Many of these problems have been discussed in recent literature and partial remedies have been suggested (Chou \& Verhaegen (1997); Lin et al. (2004); Wang \& Qin (2004); Chiuso \& Picci (2005)). Some of these problems are:

- Biased estimate for closed loop data.

- Assumption of noise-free input.

The first problem can be solved by filtering the predicted data through a frequency weighted matrix. The second one is solved by using a robust control methodology, which would cater for disturbances and noise in the system.

\footnotetext{
1 least mean square

2 filtered-x least mean squares

${ }^{3}$ multiple-input multiple-ouput
} 


\subsection{Model unfalsification}

For a true robust model free control, the system should be able to calculate an uncertainty model from the input and output data of plant. This can be done through model unfalsification. First an uncertainty model is unfalsified against the plant input and output data and then the uncertainty model $(\Delta)$ is incorporated in the controller design. Model unfalsification does not get much appeal in practice because of its high computational burden (Woodley (2001)). There are many implementations available for model unfalsificaiton (Kosut \& Anderson (1997); Agnoloni \& Mosca (2003); Tsao et al. (2003); Wodoley et al. (1999); Safonov (2003); Tsao \& Safonov (2001); Woodley et al. (1998); Cabral \& Safonov (2004)). Wang et al. suggested a direct adaptive controller based on model unfalsification with the assumption that there would be a controller in the given set that would satisfy the control requirements for a particular plant (Wang et al. (2004)). The identification of uncertainty models using model unfalsification is out of scope of this chapter.

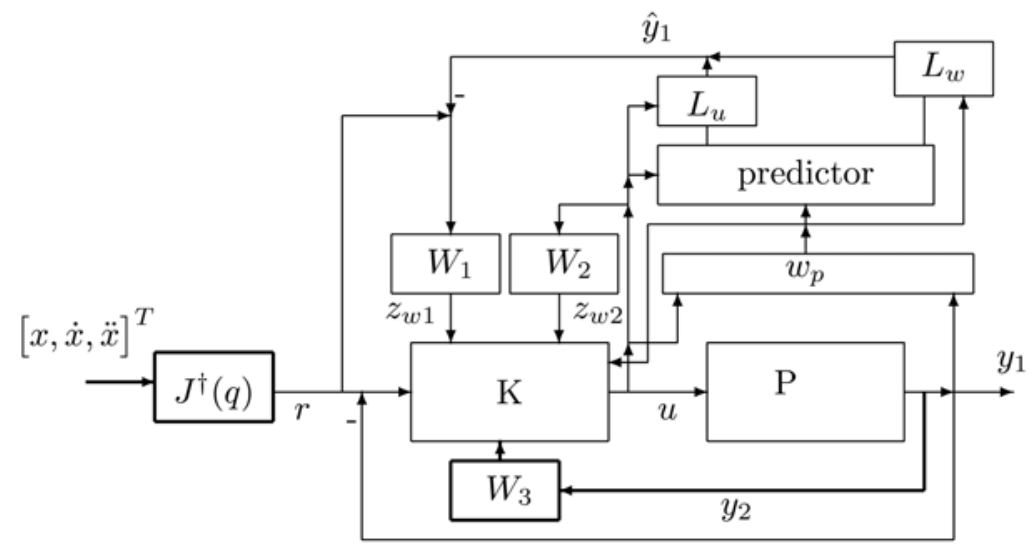

Figure 3. Model free subspace based $\mathscr{H}_{\infty}$ control. The plant transfer function $P$ is unknown and the controller transfer function $K$ is configured in realtime.

\section{Model-free subspace based dynamic control of mechanical manipulators}

The desired trajectory of end-effector is given by $[x, \dot{x}, \ddot{x}]^{\mathrm{T}}$. If the initial position of endeffector $x_{\mathrm{o}}$ and joint variables $q_{\mathrm{o}}$ is known, then using equation (2), $q, \dot{q}$ and $\ddot{q}$ can be written as

$$
\begin{gathered}
\dot{q}=J^{-1}(q) \dot{x} \\
\ddot{q}=J-1(q)(\ddot{x}-\dot{J}(q) \dot{q}) \\
\left.q_{(} t+1\right)=q_{t}+\dot{(}(q)_{t} \Delta t
\end{gathered}
$$

In case $J$ is not a square matrix, pseudo-inverse of $J$ i.e. $J^{\dagger}$ is used. Once the reference trajectory is in the joint space, model free control system which has been inspired by the work of Woodley et al. can be applied (Woodley et al. (2001b)). The cost function for this 
framework minimizes the error in joint space $[\tilde{q}, \dot{\tilde{q}}, \ddot{\tilde{q}}]^{T}$, control effort $u$, and $\dot{q}$ for the maximum value of the input $q_{r}$. Here $\tilde{q}=q-q_{r}$ and $q_{r}$ is the reference trajectory in joint space. The reason behind minimizing $\dot{q}$ is that the system becomes unstable near singularities and it becomes important that near singularities system doesn't try to achieve extremely high velocities, which could make the system unstable.

The cost function to minimized can now be written as

$$
L(\gamma)=z_{w_{1}}^{T} z_{w_{1}}+z_{w_{2}}^{T} z_{w_{2}}+z_{w_{3}}^{T} z_{w_{3}}-\gamma^{2} r^{T} r
$$

where $\gamma$ is the performance objective, and $z_{w 1}, z_{w 1}, z_{w 1}$ are weighted feedbacks of $[\tilde{q}, \dot{\tilde{q}}, \ddot{\tilde{q}}]^{T}$, $u$, and $\dot{q}$, respectively. For simplicity, lets suppose $y_{1}=[\tilde{q}, \dot{\tilde{q}}, \ddot{\tilde{q}}]^{T}, y_{2}=\dot{q}, e=\tilde{q}$, and $r=q_{r}$.

The weights are applied in frequency domain. The time domain equivalent of these weighted feedback signals can be written as

$$
\left[\begin{array}{c}
z_{w_{1}} \\
z_{w_{2}} \\
z_{w_{3}}
\end{array}\right] \triangleq\left[\begin{array}{c}
H_{1}(r-y)+\Gamma_{1}\left(x_{w_{1}}\right)_{k} \\
H_{2}(u)+\Gamma_{2}\left(x_{w_{2}}\right)_{k} \\
H_{3}\left(y_{2}\right)+\Gamma_{3}\left(x_{w_{3}}\right)_{k}
\end{array}\right],
$$

where $H_{1}$ and $H_{2}$ are lower triangular Toeplitz matrices developed from impulse responses (Markov parameters) of the discrete weighting filters, $W_{1}$ and $W_{2}$. These weights are normally assigned by the designer.

$$
\begin{array}{r}
H_{1} \triangleq\left[\begin{array}{ccccc}
D_{w 1} & 0 & 0 & \cdots & 0 \\
C_{w 1} B_{w 1} & D_{w 1} & 0 & \cdots & 0 \\
C_{w 1} A_{w 1} B_{w 1} & C_{w 1} B_{w 1} & D_{w 1} & \cdots & 0 \\
\vdots & \vdots & \vdots & \ddots & \vdots \\
C_{w 1} A_{w 1}^{i-2} B_{w 1} & C_{w 1} A_{w 1}^{i-3} B_{w 1} & C_{w 1} A_{w 1}^{i-4} B_{w 1} & \cdots & D_{w 1}
\end{array}\right] \\
H_{2} \triangleq\left[\begin{array}{ccccc}
D_{w 2} & 0 & 0 & \cdots & 0 \\
C_{w 2} B_{w 2} & D_{w 2} & 0 & \cdots & 0 \\
C_{w 2} A_{w 2} B_{w 2} & C_{w 2} B_{w 2} & D_{w 2} & \cdots & 0 \\
\vdots & \vdots & \vdots & \ddots & \vdots \\
C_{w 2} A_{w 2}^{i-2} B_{w 2} & C_{w 2} A_{w 2}^{i-3} B_{w 2} & C_{w 2} A_{w 2}^{i-4} B_{w 2} & \cdots & D_{w 2}
\end{array}\right]
\end{array}
$$

then

$$
Q_{1} \triangleq H_{1}^{\mathrm{T}} H_{1}, Q_{2} \triangleq H_{2}^{\mathrm{T}} H_{2}
$$

$\Gamma_{1}$ and $\Gamma_{2}$ are the extended observability matrices formed from the impulse responses of the weighting filters $W_{1}$ and $W_{2}$.

$$
\Gamma_{1} \triangleq\left[\begin{array}{c}
C_{w 1} \\
C_{w 1} A_{w 1} \\
\vdots \\
C_{w 1} A_{w 1}^{i-1}
\end{array}\right]
$$




$$
\Gamma_{2} \triangleq\left[\begin{array}{c}
C_{w 2} \\
C_{w 2} A_{w 2} \\
\vdots \\
C_{w 2} A_{w 2}^{i-1}
\end{array}\right]
$$

For simplicity, assume that $y=[\tilde{q}, \dot{\tilde{q}}, \ddot{\tilde{q}}]^{T}$ and $y_{2}=y_{\{3\}^{4}}$.

For system identification, suppose a plant's input and output values at discrete times are given, respectively, by

$$
\left(\left[\begin{array}{c}
u_{0} \\
u_{1} \\
\vdots \\
u_{n-1}
\end{array}\right],\left[\begin{array}{c}
y_{0} \\
y_{1} \\
\vdots \\
y_{n-1}
\end{array}\right]\right)
$$

where $u_{i} \in \Re^{m}$ and $y_{i} \in \Re^{l}$, where $m$ and $l$ are number of plant input and output signals respectively. The Hankel matrices for the past and future inputs are written as

$$
\begin{gathered}
U_{p} \triangleq\left[\begin{array}{cccc}
u_{0} & u_{1} & \cdots & u_{j-1} \\
u_{1} & u_{2} & \cdots & u_{j} \\
\vdots & \vdots & \cdots & \vdots \\
u_{i-1} & u_{i} & \cdots & u_{i+j-2}
\end{array}\right] \in \mathbb{R}^{i m \times j} \\
U_{f} \triangleq\left[\begin{array}{cccc}
u_{i} & u_{i+1} & \cdots & u_{i+j-1} \\
u_{i+1} & u_{i+2} & \cdots & u_{i+j} \\
\vdots & \vdots & \cdots & \vdots \\
u_{2 i-1} & u_{2 i} & \cdots & u_{2 i+j-2}
\end{array}\right] \in \mathbb{R}^{i m \times j}
\end{gathered}
$$

Similarly, the Hankel matrices for the past and future outputs can be written as $Y_{p} \in \Re^{i l \times j}$ and $Y_{f} \in \Re^{i l \times j}$ respectively. Hankel matrix for past outputs and inputs, $W_{p}$, could be defined as follows

$$
W_{p} \triangleq\left[\begin{array}{c}
U_{p} \\
Y_{p}
\end{array}\right]
$$

The linear least squares predictor of $Y_{\mathrm{f}}$ with given $W_{\mathrm{p}}$ and $U_{\mathrm{f}}$ can be written as Frobenius norm minimization as follows

$$
\min _{L_{w}, L_{u}}\left\|Y_{f}-\left[\begin{array}{ll}
L_{w} & L_{u}
\end{array}\right]\left[\begin{array}{l}
W_{p} \\
U_{f}
\end{array}\right]\right\|_{F}^{2}
$$

where the subspace orthogonal projections, $L_{w}$ and $L_{u}$, are calculated as

$$
\left[\begin{array}{ll}
L_{w} & L_{u}
\end{array}\right]=Y_{f}\left[\begin{array}{l}
W_{p} \\
U_{f}
\end{array}\right]^{\mathrm{T}}\left(\left[\begin{array}{l}
W_{p} \\
U_{f}
\end{array}\right]\left[\begin{array}{l}
W_{p} \\
U_{f}
\end{array}\right]^{\mathrm{T}}\right)^{\dagger}
$$

4 every third element in the array 
where $\dagger$ denotes pseudo-inverse. This solution assumes that the problem is overconstrained i.e., there are more independent equations than unknowns. If the problem is underconstrained, the pseudo-inverse cannot be computed. Future outputs can now be predicted from the past inputs, outputs, and future inputs.

$$
\left[\begin{array}{c}
\hat{y}_{k} \\
\vdots \\
\hat{y}_{k+i-1}
\end{array}\right]=L_{w}\left[\begin{array}{c}
u_{k-i} \\
\vdots \\
u_{k-1} \\
y_{k-i} \\
\vdots \\
y_{k-1}
\end{array}\right]+L_{u}\left[\begin{array}{c}
u_{k} \\
\vdots \\
u_{k+i-1}
\end{array}\right]
$$

In order to calculate $L_{w}$ and $L_{u}$, matrix decomposition methods are used. Using $Q R$ method, if

then

$$
\left[\begin{array}{c}
W_{p} \\
U_{f} \\
Y_{f}
\end{array}\right]=R^{\mathrm{T}} Q^{\mathrm{T}}=\left[\begin{array}{ccc}
R_{11} & 0 & 0 \\
R_{21} & R_{22} & 0 \\
R_{31} & R_{32} & R_{33}
\end{array}\right] Q^{\mathrm{T}}
$$

$$
\left[\begin{array}{ll}
L_{w} & L_{u}
\end{array}\right]=\left[\begin{array}{ll}
R_{31} & R_{32}
\end{array}\right]\left[\begin{array}{cc}
R_{11} & 0 \\
R_{21} & R_{22}
\end{array}\right]^{\dagger}
$$

Pseudo-inverse is normally calculated through singular value decomposition (SVD) but Woodley et al. presented another method which employs the Cholesky factorization instead of SVD (Woodley et al. (2001b)). This is computationally faster and requires less memory.

Using the strictly causal estimate of $y_{1}$ and $y_{2}$ from equation (13), we get

$$
\begin{aligned}
& \hat{y_{1}}=L_{w 1}\left(w_{p 1}\right)_{k}+L_{u 1} u \\
& \hat{y_{2}}=L_{w 2}\left(w_{p 2}\right)_{k}+L_{u 2} u
\end{aligned}
$$

Here $\hat{y}_{1}$ is the estimated value of the end effector position in the Cartesian coordinates and $\hat{y}_{2}$ is the estimated value of the joint angular velocities. From equations (7) to (16), we get

$$
\left[\begin{array}{c}
z_{w_{1}} \\
z_{w_{2}} \\
z_{w_{3}}
\end{array}\right]=\left[\begin{array}{ccccccc}
H_{1} & -H_{1} L_{u 1} & -H_{1} L_{w 1} & 0 & \Gamma_{1} & 0 & 0 \\
0 & H_{2} & 0 & 0 & 0 & \Gamma_{2} & 0 \\
0 & H_{3} L_{u 2} & 0 & H_{3} L_{w 2} & 0 & 0 & \Gamma_{3}
\end{array}\right] x
$$

where

$$
x \triangleq\left[\begin{array}{c}
r \\
u \\
w_{p 1} \\
w_{p 2} \\
\left(x_{w 1}\right)_{k} \\
\left(x_{w 2}\right)_{k} \\
\left(x_{w 3}\right)_{k}
\end{array}\right]
$$


Substituting equation (7) into (6) and (17) produces the objective

$$
\min _{u} \sup _{r} x^{T} W x \leq 0
$$

where $W$ is given as follows;

$W=\left[\begin{array}{ccccccc}Q_{1}-\gamma^{2} I & -Q_{1} L_{u 1} & -Q_{1} L_{w 1} & 0 & H_{1}^{T} \Gamma_{1} & 0 & 0 \\ -L_{u 1}^{T} Q_{1} & L_{u 1}^{T} Q_{1} L_{u 1}+Q_{2}+L_{u 2}^{T} Q_{3} L_{u 2} & L_{u 1}^{T} Q_{1} L_{w 1} & L_{u 2}^{T} Q_{3} L_{w 2} & -L_{u 1}^{T} H_{1}^{T} \Gamma_{1} & H_{2}^{T} \Gamma_{2} & H_{3}^{T} \Gamma_{3} \\ \vdots & \vdots & \vdots & \vdots & \vdots & \vdots & \vdots \\ \cdots & \cdots & \cdots & \cdots & \cdots & \cdots & \Gamma_{3}^{T} \Gamma_{3}\end{array}\right]$

Differentiating (19) with respect to $[r u]^{T}$ and equating to zero produces the following;

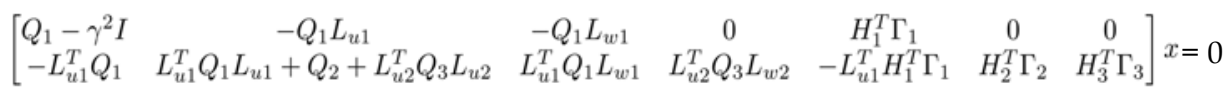

The linear system in (20) can be re-arranged as follows;

$$
\begin{aligned}
& {\left[\begin{array}{cc}
Q_{1}-\gamma^{2} I & -Q_{1} L_{u 1} \\
-L_{u 1}^{T} Q_{1} & L_{u 1}^{T} Q_{1} L_{u 1}+Q_{2}+L_{u 2}^{T} Q_{3} L_{u 2}
\end{array}\right]\left[\begin{array}{l}
r \\
u
\end{array}\right]}
\end{aligned}
$$

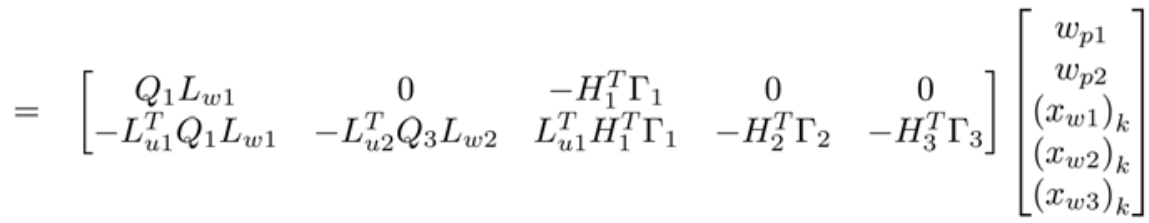

Differentiating once again with respect to $[r u]^{T}$ suggests the following;

$$
H \triangleq \frac{\partial^{2} J(\gamma)}{\partial\left[\begin{array}{l}
r \\
u
\end{array}\right]^{2}}=\left[\begin{array}{cc}
Q_{1}-\gamma^{2} I & -Q_{1} L_{u 1} \\
-L_{u 1}^{T} Q_{1} & L_{u 1}^{T} Q_{1} L_{u 1}+Q_{2}+L_{u 2}^{T} Q_{3} L_{u 2}
\end{array}\right]
$$

Schur decomposition offers the following definition;

$$
\left[\begin{array}{cc}
A_{1} & A_{2} \\
A_{2}^{T} & A_{3}
\end{array}\right]=\left[\begin{array}{cc}
I & A_{2} A_{2}^{-1} \\
0 & I
\end{array}\right]\left[\begin{array}{cc}
A_{1}-A_{2} A_{3}^{-1} A_{2}^{T} & 0 \\
0 & A_{3}
\end{array}\right]\left[\begin{array}{cc}
I & 0 \\
A_{3}^{-1} A_{2}^{T} & I
\end{array}\right]
$$

Since $Q_{1}=Q_{1}^{T}$ and $Q_{1}$ is positive definite, it could be concluded that $A_{3}>0$ which satisfies the saddle condition (Woodley (2001)). As $A_{3} \in \Re^{i m \times i m}$ and $r \in \Re^{i m}$, the condition for worst case input reference signal can be stipulated by the following inequality;

$$
A_{1}-A_{2} A_{3}^{-1} A_{2}^{T}<0
$$

Matching the definitions in (23) and (24) to the mathematical aspects of the model-free control introduced above, the following could be stated; 


$$
Q_{1}-Q_{1} L_{u 1}\left(L_{u 1}^{T} Q_{1} L_{u 1}+Q_{2}+L_{u 2}^{T} Q_{3} L_{u 2}\right)^{-1} L_{u 1}^{T} Q_{1}<\gamma^{2} I
$$

which can be written as;

$$
\gamma>\sqrt{\bar{\lambda}\left[Q_{1}-Q_{1} L_{u 1}\left(L_{u 1}^{T} Q_{1} L_{u 1}+Q_{2}+L_{u 2}^{T} Q_{3} L_{u 2}\right)^{-1} L_{u 1}^{T} Q_{1}\right]} \equiv \gamma_{\min }
$$

To calculate the optimum controller outputs, multiply (21) with [0 I].

$$
\begin{aligned}
& u_{\text {opt }}=\left[\begin{array}{ll}
0 & I
\end{array}\right]\left[\begin{array}{cc}
Q_{1}-\gamma^{2} I & -Q_{1} L_{u 1} \\
-L_{u 1}^{T} Q_{1} & L_{u 1}^{T} Q_{1} L_{u 1}+Q_{2}+L_{u 2}^{T} Q_{3} L_{u 2}
\end{array}\right]^{-1}
\end{aligned}
$$

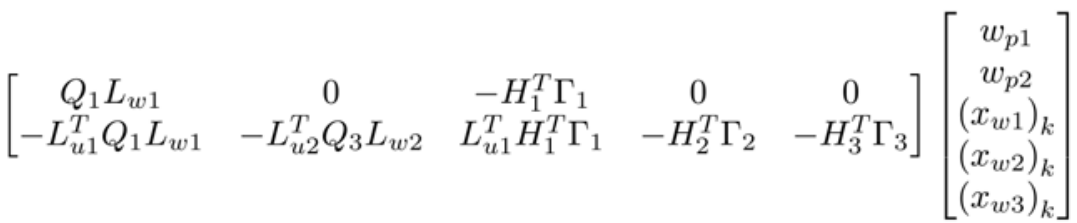

\subsubsection{Uncertainties}

For a robust system, it is important that uncertainties are accounted for. Most uncertainties in a plant are hard to model. Figures (4) and (5) show the general layout of plant models with uncertainties in multiplicative and additive configurations, respectively. Woodley calculated $\gamma_{\min }$ for different configurations of uncertainties in model-free control designs (Woodley (2001)). But the real challenge is to find the uncertainty block $\Delta$ through techniques like model unfalsification. A true robust system calculates $\Delta$ in realtime.

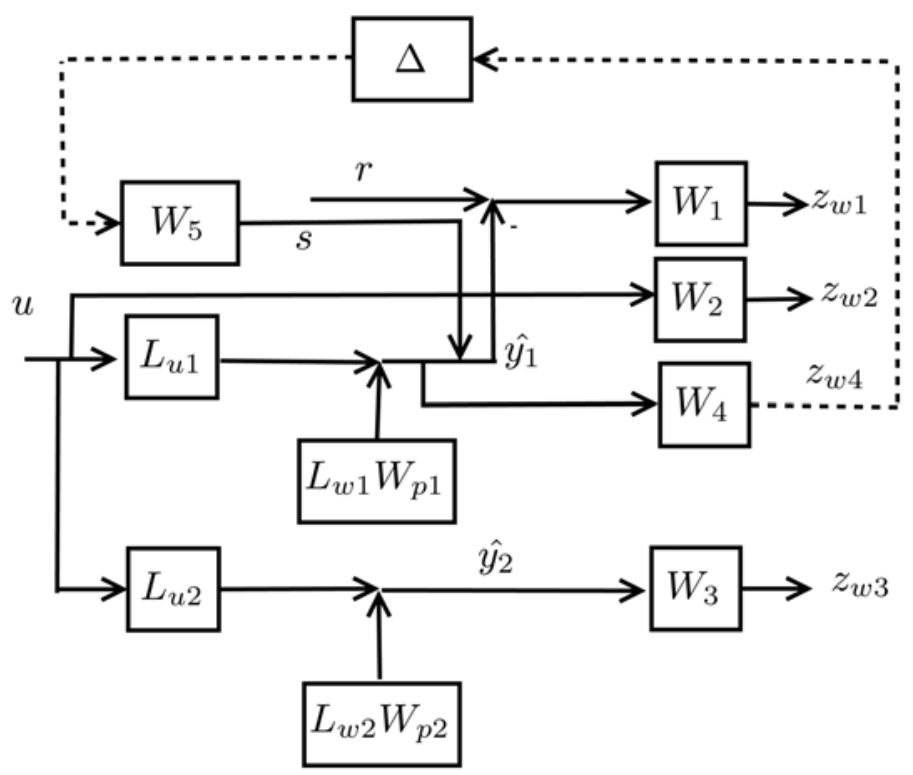

Figure 4. Plant with multiplicative uncertainties for robust $\mathscr{H}_{\infty}$ control design 


\section{Simulation}

For a complete identification $13 n$ parameters are to be identified (Khalil and Dombre (2004)). In model free framework, these parameters are not available explicitly. The prediction horizon should be two to three times the expected order of the system. From this rough estimate, prediction horizon as 30 is selected for a two joint planar manipulator. On one of the coordinate, a square signal and on the other one, a sinusoidal signal is given. The response of the end effector along with the performance objective $\gamma$ is given in the figure (6). One of the benefit of using subspace identification is the property of Hankel matrix that allows to concatenate the data from a previous session.

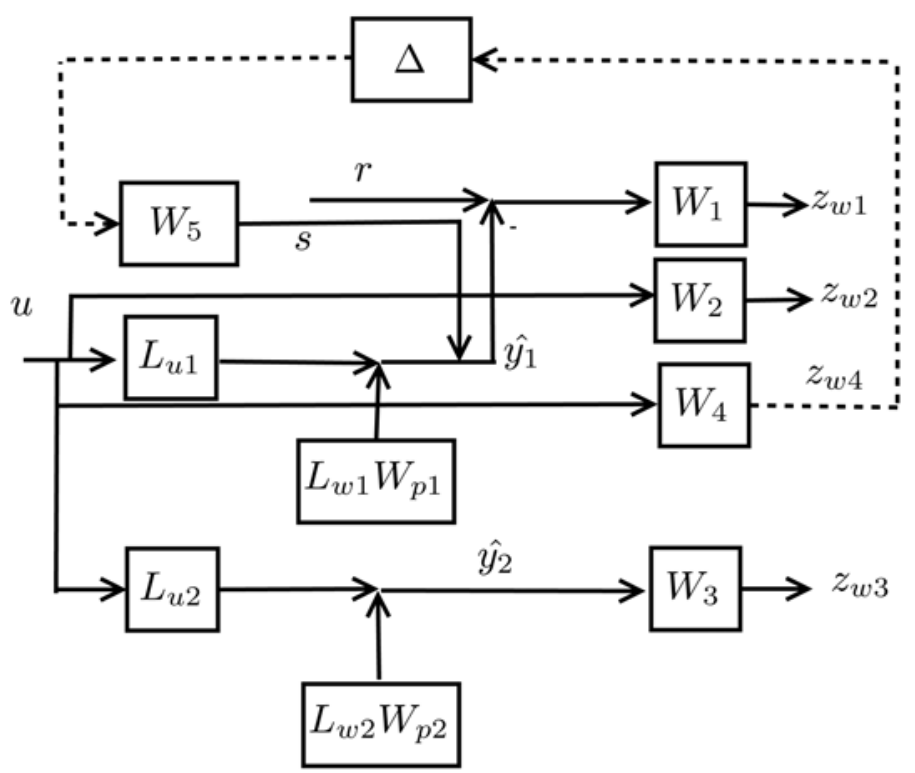

Figure 5. Plant with additive uncertainties for robust $\mathscr{H}_{\infty}$ control design

\section{Conclusion}

The proposed framework provides solution to inverse dynamics, parameter identification and robust control of mechanical manipulators in an elegant way. The fastest way to calculate pseudo-inverse is through Cholesky/SVD factorization (Golub \& Loan (1996)). Its complexity is $O\left(i j+i^{3}\right)$, where $i$ is the prediction horizon and $j$ is number of prediction problems in a Hankel matrix. The computational burden becomes significant when a robot has large number of links and it has 6 degrees of freedom i.e. $13 \ln$, where $l$ is a arbitrary natural number from 1 to 5 as a safety factor in prediction and $n$ is the number of joints of manipulator. Calculations required to predict a predictor for a six joint robot with six degrees of freedom with safety margin of 2 is 3796416 . This number is not so big for modern computers but increasing the number of joints will increase the complexity exponentially. 
Reference signals vs. plant outputs

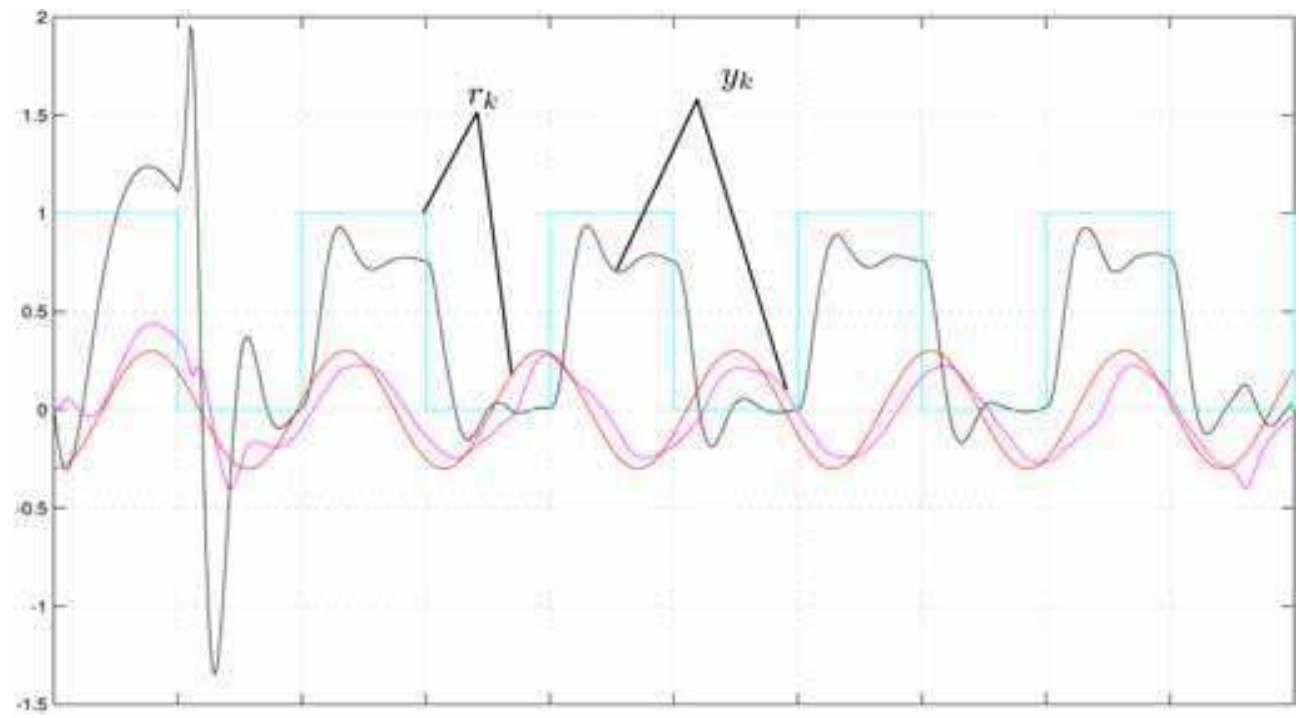

$\gamma$ as a function of time

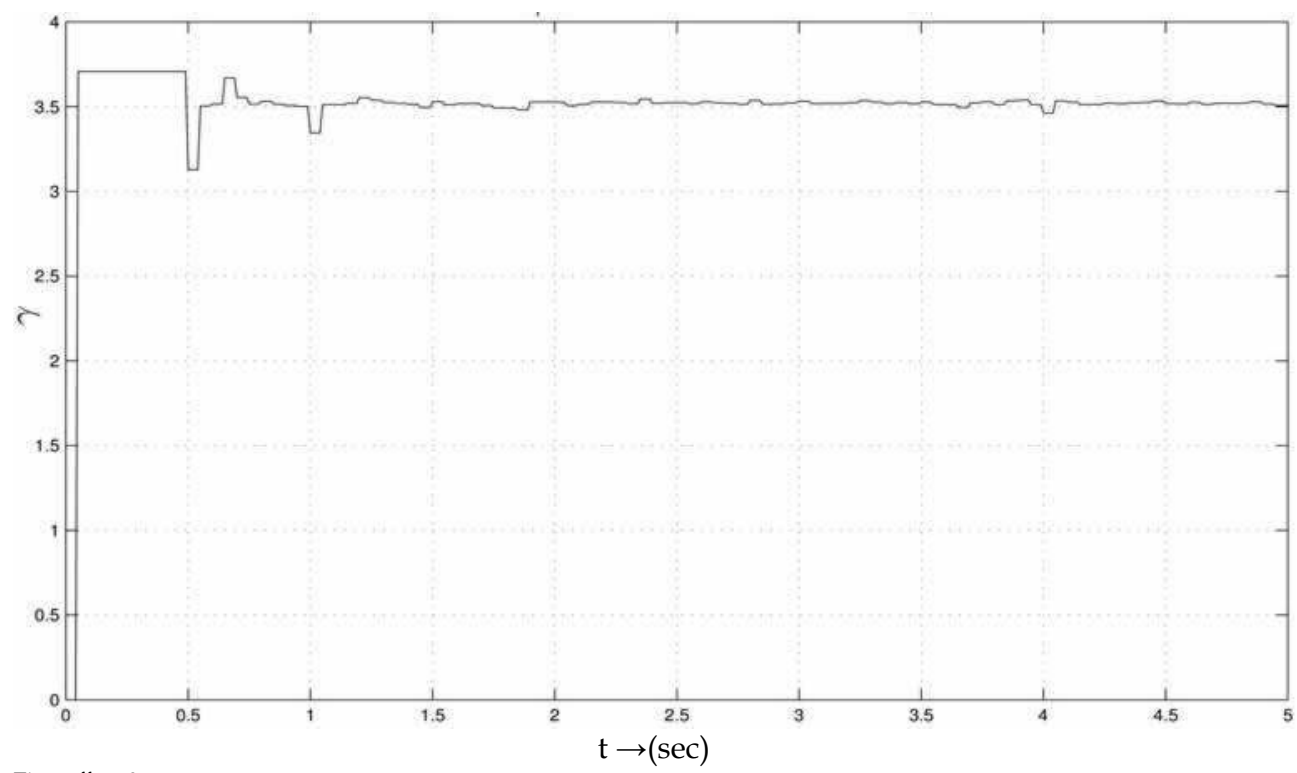

Time offset: 0

Figure 6. Response of a planar robot with two rotary joints. In this particular experiment, $W_{p}$ is initialized with null matrix. The performance is given by $\gamma$, which converges to a constant value when the input is consistent. 


\section{References}

Agnoloni, T. and Mosca, E. (2003). Controller falsification based on multiple models. International Journal of Adaptive Control and Signal Processing, 17:163-177.

Arimoto, S. (1995). Fundamental problems of robot control: Parti, innovations in the realm of robot servo-loops. Robotica, 13:19-27.

Athans, M. (1999). Crisp control is always better than fuzzy feedback control. EUFIT t'99 debate with Prof. L.A. Zadeh, Aachen, Germany.

Boyd, J. E. and Little, J. J. (2000). Phase in model-free perception of gait. In HUMO '00: Proceedings of the Workshop on Human Motion (HUMO'00), page 3, Washington, DC, USA. IEEE Computer Society.

Caballero, R., Armada, M. A., and Akinfiev, T. (2004). Robust cascade controller for nonlinearly actuated biped robots: experimental evaluation. International Journal of Robotics Research, 23(10/11):1075-1095.

Cabral, F. B. and Safonov, M. G. (2004). Unfalsified model reference adaptive control using the ellipsoid algorithm. International Journal of Adaptive Control and Signal Processing, 18(8):683-696.

Cheng, G. (2004). Model-free adaptive (mfa) control. Computing \& Control Engineering Journal, 15(3):28-33.

Chiuso, A. and Picci, G. (2005). Consistency analysis of some closed-loop subspace identification methods. Automatica, 41(3):377-391.

Chou, C. T. and Verhaegen, M. (1997). Subspace algorithms for the identification of multivariable dynamic errors-in-variables models. Automatica, 33:1857-1869.

Date, P. and Lanzon, A. (2004). A combined iterative scheme for identification and control redesigns. International Journal of Adaptive Control and Signal Processing, 18(8):629_ 644.

Favoreel, W., Moor, B. D., Gevers, M., and Overschee, P. V. (1998). Model-free subspacebased LQG-design. Technical report, Katholieke Universiteit Leuven.

Favoreel, W., Moor, B. D., Gevers, M., and Overschee, P. V. (1999a). Closed loop model-free subspace-based LQG-design. In Proceedings of the IEEE Mediterranean Conference on Control and Automation, Haifa, Israel.

Favoreel, W., Moor, B. D., and Overschee, P. V. (1999b). Model-free subspace-based LQGdesign. In Proceedings of the American Control Conference, pages 3372-3376.

Golub, G. H. and Loan, C. F. V. (1996). Matrix Computations. The Johns Hopkins University Press.

Grimble, M. J. (1998). Multi-step $\mathscr{H}_{\infty}$ generalized predictive control. Dynamics and Control, 8(4):303-339.

Haddad, W. M., Hayakawa, T., and and, J. M. B. (2003). Adaptive control for nonnegative and compartmental dynamical systems with applications to general anesthesia. International Journal of Adaptive Control and Signal Processing, 17(3): 209- 235.

Hayakawa, T., Haddad, W. M., and Leonessa, A. (2004). A Lyapunov-based adaptive control framework for discrete-time non-linear systems with exogenous disturbances. International Journal of Control, 77(3): 250-263.

Hsu, E., Pulli, K., and Popovi'c, J. (2005). Style translation for human motion. ACM Transactions on Graphics (TOG), 24(3): 1082-1089. 
Kelly, R. (1993). Comments on adaptive pd controller for robot manipulators. IEEE Trans. Robot. Automat., 9: 117-119.

Kelly, R. (1997). Pd control with desired gravity compensation of robotic manipulators: A review. Int. J. Robot. Res., 16(5): 660-672.

Khalil, W. and Dombre, E. (2004). Modeling, Identification and Control of Robots. Kogan Page Science.

Kim, D., Kim, N.-H., Seo, S.-J., and Park, G.-T. (2004). Fuzzy Modeling of Zero Moment Point Trajectory for a Biped Walking Robot. Lecture Notes in Computer Science. SpringerVerlag $\mathrm{GmbH}, 3214$ edition.

Kim, J. O., Lee, B. R., Chung, C. H., Hwang, J., and Lee, W. (2003). The Inductive Inverse Kinematics Algorithm to Manipulate the Posture of an Articulated Body. Lecture Notes in Computer Science. Springer-Verlag $\mathrm{GmbH}, 2657$ edition.

Kosut, R. L. and Anderson, B. D. O. (1997). Uncertainty model unfalsification. In Proceedings of the 36th IEEE Conference on Decision and Control, volume 1, pages 163-168, San Diego, CA.

Laib, A. (2000). Adaptive output regulation of robot manipulators under actuator constraints. IEEE Trans. Robot. Automat., 16:29-35.

Lin, W., Qin, S. J., and Ljung, L. (2004). A framework for closed-loop subspace identification with innovation estimation. Technical Report 2004-07, Department of Chemical Engineering, The University of Texas at Austin, Austin, TX 78712, USA and Linköping University, SE-581 83 Linköping, Sweden.

Ljung, L. (1999). System identification: theory for the user. Prentice-Hall, Upper Saddle River, NJ, USA.

Lohar, F. A. (2000). $\mathscr{H}_{\infty}$ and $\mu$-synthesis for full control of helicopter in hover. In 38th Aerospace Sciences Meeting and Exhibit, Reno, NV. American Institute of Aeronautics and Astronautics.

Norton, J. P. (1986). Introduction to Identification. Academic Press.

Overschee, P. V. and Moor, B. D. (1996). Subspace Identificiation for Linear Systems. Kluwer Academic Publishers.

Safonov, M. G. (2003). Recent advances in robust control theory. In AIAA Guidance, Navigation and Control Conference and Exhibit, volume 4, Austin, TX.

Sapio, V. D. and Khatib, O. (2005). Operational space control of multibody systems with explicit holonomic constraints. In Proceedings of the 2005 IEEE International Conference on Robotics and Automation.

Sayyar-Rodsari, B., How, J. P., Hassibi, B., and Carrier, A. (1998). An $\mathscr{H}_{\infty}$ optimal alternative to the fxlms algorithm. In Proceedings of the American Control Conference, pages 11161121.

Sciavicco, L. and Siciliano, B. (2000). Modelling and Control of Robot Manipulators. Springer, 2nd edition.

Takegaki, M. and Arimoto, S. (1981). A new feedback method for dynamic control of manipulators. ASME J. Dyn. Syst., Meas., Control, 102:119-125.

Tomei, P. (1991). Adaptive pd controller for robot manipulators. IEEE Trans. Robot. Automat., 7:565-570. 
Tsao, T.-C., Brozenec, T., and Safonov, M. G. (2003). Unfalsified adaptive spacecraft attitude control. In AIAA Guidance, Navigation, and Control Conference and Exhibit, Austin, TX.

Tsao, T.-C. and Safonov, M. G. (2001). Unfalsified direct adaptive control of a twolink robot arm. International Journal of Adaptive Control and Signal Processing, 15(3):319-334.

Tse, J., Bentsman, J., and Miller, N. (1993). Properties of the self-tuning minimax predictive control (MPC). In Proceedings of the 1993 American Control Conference, pages 17211725 .

Wang, J. and Qin, S. J. (2004). A new deterministic-stochastic subspace identification method using principal component analysis. Technical report, Department of Chemical Engineering, The University of Texas at Austin.

Wang, R., Stefanovic, M., and Safonov, M. G. (2004). Unfalsified direct adaptive control using multiple controllers. In AIAA Guidance, Navigation, and Control Conference and Exhibit, pages 1-16, RI, USA.

Wen, J., Kreutz-Delgado, K., and Bayard, D. (1992). Lyapunov function-based control laws for revolute robot arms. IEEE Trans. Automat. Contr., 37:231-237.

Wernholt, E. (2004). On Multivariable and Nonlinear Identification of Industrial Robots. PhD thesis, Department of Electrical Engineering, Link“oping University, SE-581 83 Linköping, Sweden.

Widrow, B. and Stearns, S. D. (1985). Adaptive Signal Processing. Prentice-Hall.

Widrow, B. and Walach, E. (1994). Adaptive Inverse Control. Prentice-Hall.

Wilson, E. (1997). Adaptive profile optimization for the electric arc furnace. In Steel Technology International, pages 140-145.

Wodoley, B., How, J., and Kosut, R. (1999). Direct unfalsified controller design - solution via convex optimization. In Proceedings of the 1999 American Control Conference, volume 5, pages 3302-3306.

Wolkotte, P. T. (2003). Modelling human locomotion. Technical report, Institute of Electronic Systems, Aalborg University.

Woodley, B., How, J., and Kosut, R. (2001a). Model free subspace based $\mathscr{H}_{\infty}$ control. In Proceedings of the 2001 American Control Conference, volume 4, pages 2712- 2717.

Woodley, B., Kosut, R., and How, J. (1998). Uncertainty model unfalsification with simulation. In Proceedings of the 1998 American Control Conference, volume 5, pages 2754-2755.

Woodley, B. R. (2001). Model free subspace based $\mathscr{H}_{\infty}$ control. PhD thesis, Department of Electrical Engineering, Stanford University.

Woodley, B. R., How, J. P., and Kosut, R. L. (2001b). Subspace based direct adaptive $\mathscr{H}_{\infty}$ control. International Journal of Adaptive Control and Signal Processing, 15(5):535-561.

Xie, M. (2003). Fundamentals of Robotics, volume 54 of Machine perception and artificial intelligence. World Scientific.

Zhang, Y., Tian, H., Wang, Q., and Qiang, W. (2000). Servo control in joint space of biped robot using nonlinear $\mathscr{H}_{\infty}$ strategy. In Jiang, D. and Wang, A., editors, Proceedings of SPIE, International Conference on Sensors and Control Techniques (ICSC 2000), volume 4077, pages 386-391. 
Zhao, H. and Bentsman, J. (1999). Multivariable $\mathscr{H}_{\infty}$ predictive control based on minimax predictor. In Proceedings of the IEEE Conference on Decision and Control, volume 4, pages 3699-3705. 


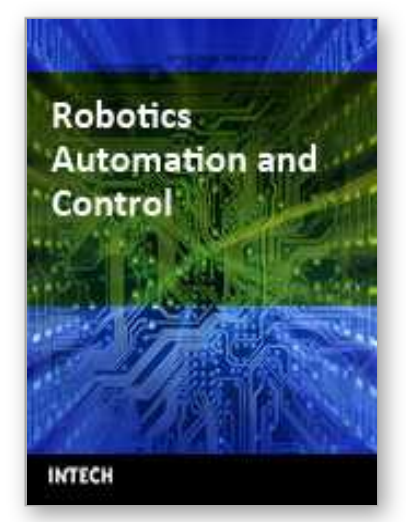

\author{
Robotics Automation and Control \\ Edited by Pavla Pecherkova, Miroslav Flidr and Jindrich Dunik
}

ISBN 978-953-7619-18-3

Hard cover, 494 pages

Publisher InTech

Published online 01, October, 2008

Published in print edition October, 2008

This book was conceived as a gathering place of new ideas from academia, industry, research and practice in the fields of robotics, automation and control. The aim of the book was to point out interactions among various fields of interests in spite of diversity and narrow specializations which prevail in the current research. The common denominator of all included chapters appears to be a synergy of various specializations. This synergy yields deeper understanding of the treated problems. Each new approach applied to a particular problem can enrich and inspire improvements of already established approaches to the problem.

\title{
How to reference
}

In order to correctly reference this scholarly work, feel free to copy and paste the following:

Muhammad Saad Saleem and Ibrahim A. Sultan (2008). Model-free Subspace Based Dynamic Control of Mechanical Manipulators, Robotics Automation and Control, Pavla Pecherkova, Miroslav Flidr and Jindrich Dunik (Ed.), ISBN: 978-953-7619-18-3, InTech, Available from:

http://www.intechopen.com/books/robotics_automation_and_control/model-

free_subspace_based_dynamic_control_of_mechanical_manipulators

\section{INTECH}

open science | open minds

\section{InTech Europe}

University Campus STeP Ri

Slavka Krautzeka 83/A

51000 Rijeka, Croatia

Phone: +385 (51) 770447

Fax: +385 (51) 686166

www.intechopen.com

\section{InTech China}

Unit 405, Office Block, Hotel Equatorial Shanghai

No.65, Yan An Road (West), Shanghai, 200040, China 中国上海市延安西路65号上海国际贵都大饭店办公楼 405 单元 Phone: +86-21-62489820

Fax: $+86-21-62489821$ 
(C) 2008 The Author(s). Licensee IntechOpen. This chapter is distributed under the terms of the Creative Commons Attribution-NonCommercialShareAlike-3.0 License, which permits use, distribution and reproduction for non-commercial purposes, provided the original is properly cited and derivative works building on this content are distributed under the same license. 